\title{
Impact and Challenges of Ethics in Echocardiography - A Non-Invasive Procedure
}

\author{
Sujhithra Appan Ramanujam¹, Chokkalingam Meyyappan², Jayanthi Sivasubramaniam³, \\ Sanjay Andrew Rajaratnam ${ }^{4}$, Danis Vijay Devaraj ${ }^{5}$
}

${ }^{1}$ Department of Allied Health Sciences, Chettinad Hospital and Research Institute (CHRI), Chettinad Academy of Research and Education, Kelambakkam, Tamilnadu, India. ${ }^{2}$ Department of Cardiology, Chettinad Hospital and Research Institute (CHRI), Chettinad Academy of Research and Education, Kelambakkam, Tamilnadu, India. ${ }^{3}$ Department of Microbiology, Panimalar Medical College Hospital and Research Institute, Varadhrajapuram, Poonamallee, Tamilnadu, India. ${ }^{4}$ Department of Physiology, Chettinad

Hospital and Research Institute (CHRI), Chettinad Academy of Research and Education, Kelambakkam, Tamilnadu, India. ${ }^{5}$ Department of Microbiology, Chettinad Hospital and Research Institute (CHRI), Chettinad Academy of Research and Education, Kelambakkam, Tamilnadu, India.

\section{ABSTRACT}

\section{BACKGROUND}

Increasingly sophisticated types of equipment are needed for the evaluation of cardiac function and structure. An invaluable diagnostic tool with broadening applications, which is also portable and cost-effective in medical and surgical practice is echocardiography. The purpose of this review is to derive the ethical constraints, ethical practices and challenges in the field of echocardiography, when practicing echocardiography in a clinical setup.

Ethics in echocardiography involves the "transfer of value" among the physicians, hospitals and patients. Altruism, integrity, responsibility and respect are the four pillars of professionalism. These are to be strictly followed with the patients, trainees and colleagues. Four basic ethics associated in the patient care are patient preferences, quality of life, medical indications and contextual features.

The American College of Cardiology Foundation and ASE's (American Society of Echocardiography) appropriate use criteria insist upon the periodic review in every echocardiography laboratory. Other issues include a mechanism for timelines of test performance and test selection. Ethics in modern practice, so far known to us, are only the tip of the iceberg. Qualified sonographers and physicians should work by ensuring the ethical issues in the diagnostic field echocardiography.

\section{KEY WORDS}

Ethics, Echocardiography, Indications, Preferences, Challenges
Corresponding Author: Dr. Jayanthi Sivasubramaniam, Professor, Department of Microbiology, Panimalar Medical College Hospital \& Research Institute, Varadhrajapuram, Poonamallee - 600123, Chennai, Tamil Nadu, India. E-mail: jayanthitanmicro@gmail.com

DOI: $10.14260 /$ jemds/2021/22

How to Cite This Article:

Ramanujam SA, Meyyappan C, Sivasubramaniam J, et al. Impact and challenges of ethics in echocardiography: a non- invasive procedure. J Evolution Med Dent Sci 2021;10(02):107-110, DOI: 10.14260/jemds/2021/22

Submission 15-09-2019,

Peer Review 14-11-2020,

Acceptance 20-11-2020,

Published 11-01-2021.

Copyright (C) 2021 Sujhithra Appan Ramanujam et al. This is an open access article distributed under Creative Commons Attribution License [Attribution 4.0 International (CC BY 4.0)] 


\section{BACKGROUND}

Acquisition of skill for performing the examination, and increasingly sophisticated types of equipment, are needed for evaluating the effective functioning of cardiac structures. ${ }^{1} \mathrm{An}$ invaluable diagnostic tool with broad applications, which is portable and cost effective in medical and surgical practice is echocardiography. ${ }^{2}$

Growing professional and educational demands recommends excellence and stands for quality which is under the vision of ASE. ${ }^{1}$ The cost-effective, most versatile imaging technique is used to assess cardiovascular disease, comprehensive assessment and haemodynamic status of patients with high risk. The decision-making process, in the emergency setting as well as in the patient management of cardiovascular emergencies algorithms, has included echocardiography as a primary tool for evaluation. ${ }^{3}$ In this review paper, we aim to propagate the ethical constraints, ethical practices and challenges in the field of echocardiography, better knowledge when practising echocardiography in the clinical setup.

\section{Evolving Ethics in Echocardiography}

Medical ethics is termed as a combination of moral values and principles used to judge research, medical practice and education. History tells us that guidelines on a medical practitioner's duty, conduct and moral responsibility have been thoroughly evaluated and that the first code of medical ethics called "Formula Comitis Archiatrorum"Ostrogothic King Theodoric the Great back in the $5^{\text {th }}$ century. ${ }^{4}$

Historically Western people have often practiced medical ethics in the form of Hippocratic Oath. Early in the $18^{\text {th }}$ century, ethics in modern medicine were started, and the book on medical ethics was written by Thomas Percival, coined the medical ethics and medical jurisprudence terminology. American Medical Association also came up with a code of ethics in medical practice which was primarily based on Thomas Percival book. From being more eccentric and uncompetitive, medical ethics endured a dramatic shift due to more liberal thinking and considering procedural justice has developed into what is called bioethics. ${ }^{4}$

We should be thankful to philosophers for a specific deontological strategy which numerous healthcare professionals, students have adopted today, an ethical lifebelt with four bioethics principles. ${ }^{5}$

Four principles are beneficence, non-maleficence, respect for autonomy and justice is a convenient checklist of the ethical perspectives to be covered when considering morally objectionable questions or situations. "What will do good, avoid harm, or at least do more good than harm for the patient concerned?" Is it taking the wishes of the persons involved seriously? "What is the most equitable, not only for this patient or patient group but also for others and society? ${ }^{6}$

There are now countless ethically problematic clinical cases examined in terms of this conceptual framework. The issue about this framework is that, as advertised the four principles are each prima facie; in other words, it is a principle that must be accompanied, given this does not conflict with the other principle of equal importance. Moreover, there is a rub. ${ }^{5}$

For example, more good than harm will be done for the patient through immediate treatment as stated by beneficence and non-maleficence: But the patient refuses treatment, is competent and therefore cannot be administered treatment under respect for autonomy. Justice is no help if the refusal of the patient severely jeopardizes the vital interests of any other person. In certain statements, the problem with the principles is that they do not provide a decision procedure for conflict resolution or arriving at realistic conclusions. ${ }^{5}$

1. US Department of Health and Human Services made publicly available a list of payments and Centers for Medicare and Medicaid Services hosted a searchable website in order to "transfer of value" among the physicians, hospitals and patients. Open Payments system established the Patient Protection and Affordable Care Act of $2009-2010$ to overcome the difficulties. ${ }^{7}$

2. Quality is a commitment to public concern and depends on the tenet of medical professionalism. Improvement in quality metrics is needed to set a benchmark performance. Documentation due to lapses in quality was demonstrated in Harlem Hospital in the year of 2010 with abnormal findings (unread 4000 Echocardiograms). Because of poor interpretation and poor technical quality, "the sceptical cardiologist" recommended the "advanced training and competence" among the echocardiographers and registered sonographers. ${ }^{8}$

3. The ethical and humanitarian practice of Medicine was demonstrated as the four pillars of professionalism by Dr. Kerber. Altruism, integrity, responsibility and respect are illustrated and to handle them with the patients, trainees and colleagues. ${ }^{9}$

\section{Ethical Constraints Associated with Patient Care 10,11}

Four basic ethics associated with patient care are as follows-

1. Medical Indications: Consists of diagnosis, treatment and prognosis options.

2. Patient Preferences: Includes patient goals, autonomy, patient values.

3. Quality of Life: Consists of third party's (except patient's) assessment of the quality of benefits and treatment and burdens of illness.

4. Contextual Features: Public health impacts, religious, financial considerations, cultural, family factors and conflicts of interest.

\section{Recommendations for Echocardiography 12,13}

1. The American College of Cardiology Foundation (ACCF) and ASE appropriate use of criteria insisting upon the periodic review in every echocardiography laboratory. It can be:

- Review with all interpreting physicians and sonographers.

- Quality measures about echocardiography at a national level and measures related to imaging.

- The aim is to educate appropriate use of criteria.

- Awareness of clinical scenarios where the echo is over and underused.

- Echocardiographic recommendations are illustrated in ASE, but these criteria do not intend to be all-inclusive always.

2. Laboratories should verify whether the echocardiography is ordered appropriately or not. A systematic review will 
enable appropriate and inappropriate indications to be distinguished along with updated national norms and periodic feedback for the right clinical practices from the ordering physicians.

The above recommendations provide a framework for the assessment of practice and improvement in the quality metrics with the appropriate echocardiographic exams among the qualifiers.

\section{Other Issues ${ }^{14}$}

1. Access: Mechanism for timelines of test performance, i.e., tracking of echocardiographic studies wait times (outpatient and inpatient).

2. Test selection: Test components are as follows:

-Correct components of a transthoracic and trans oesophageal echocardiographic study (i.e., doppler, rest, stress, M-mode, 3D, assessment of shunt)

-Optimum stress modality (exercise or pharmacologic)

-Characteristics of the patient.

\section{Echocardiography Laboratories Should Have ${ }^{2}$}

1. Staff members who understand the criteria to be used appropriately.

2. Reduce the inappropriate referrals (referring physicians ethical education is mandatory).

3. Procedure selection and application of appropriate criteria have to be taken care.

\section{Patient Selection ${ }^{12,13}$}

Avoid misuse, underuse and overuse of echocardiography by selecting the patients appropriately. The procedure should be undertaken when the patient benefit is high. It should improve patient selection by minimizing inappropriate requests. Individual patient considerations and physician judgment should adhere to appropriate use of criteria.

\section{Ethics in Request ${ }^{14}$}

Patient information should have adequate clinical data and treatment with appropriateness. In charge person (sonographer) should track upon inappropriate request of the patients which has been ordered.

\section{Revised Scope of Practice ${ }^{15}$}

Representatives from 16 different organizations in May 2013 revised the scope of collaborative practice and the process of ideas exchange in both standard clinical practice and scope of practices.

\footnotetext{
Clinical Standards of Diagnostic Sonography ${ }^{15}$ Standards which includes section,

1.1 Patient Information assessments and evaluation,

1.2 Patient education and communication,

1.3 Analysis and determination of protocol for the diagnostic examination,
}

1.4 Implementation of the protocol,

1.5 Evaluation of the diagnostic examination results,

1.6 Documentation.

2.1 Implement quality improvement programs,

2.2 Quality of care.

3.1 Self-assessment, education.

3.2 Collaboration.

3.3 Ethics.

These are intended to: reflect the expected performance and behaviour levels of medical sonographers in the clinical practice. Clinical areas or individual specialties may expand or optimize these general standards, but do not limit them according to their particular practice requirements.

Among these above said standards, we have mentioned here only the documentation and ethics in detail.

\section{Standard-Documentation ${ }^{5}$}

Documentation should be clear and accurate and required for accuracy of care, quality assurance and continuity of care. The medical sonographer:

- Provides concise, timely, accurate and proper documentation.

- $\quad$ Provides a written or summary of the findings and refers to the supervising physician as appropriate.

\section{Standard-Ethics 15}

All decisions would be made and actions taken on patient's behalf adhere to ethical standards.

The diagnostic medical sonographer:

- Adheres to accepted professional, ethical standards.

- Is accountable for decisions and professional judgments.

- Provides patient care with equal respect for all.

- $\quad$ Respects and promotes patient rights to respect patient dignity and needs, acts as advocates for patients.

- Except in educational activities, does not perform sonographic procedures without medical indication.

The ethics which we are discussing are only the 'Tip of the Iceberg'. Many will arise in the future, which will provide permanent content for the future domain of discussion.

\section{Ethics with Modern Technology ${ }^{9}$}

Improvements in accuracy, efficiency and standardization are the promises of Artificial Intelligence (AI). A component of artificial intelligence, machine learning will be able to generate a report in the future. One day, robot-assisted scanning captures all the views from the standard echo-windows without the influence of human direction.

\section{Near Challenges will be Faced on Upcoming Technology}

Due to technology improvement, despite the clinical presentation, machines will be able to analyze finding, but they can never fulfil the discussion with a referring clinician in the clinical context. ${ }^{9}$

In order to get changes in health care in the future, many have recommended collaborative practice and the process of 
ideas exchange in both standard clinical practice and scope of practices. 15

\section{CONCLUSIONS}

Without the interference of the human element, we cannot get an accurate result even with high end technologies. As a team, qualified sonographers and physicians should work together in order to harness the full potential of echocardiography in the diagnostic field. By considering the above ethical standards which should be practiced, it would enable us to perform better with the patients and also in the emergency set up, by delivering better health care in the field of diagnostic medical echocardiography.

Data sharing statement provided by the authors is available with the full text of this article at jemds.com.

All Authors received grants from Chettinad Academy of Research and Education.

We thank Prof. Dr. R. Murugesan Director-Research (Chettinad Academy of Research and Education) and Dr. Priyadarshini Shanmugam HOD, Department of Microbiology, (Chettinad Hospital and Research Institute) for valuable suggestions and support.

\section{REFERENCES}

[1] Bierig SM, Ehler D, Knoll ML, et al. American society of echocardiography minimum standards for the cardiac sonographer: a position paper. J Am Soc Echocardiogr 2006;19(5):471-4.

[2] Picard MH, Adams D, Bierig SM, et al. American society of echocardiography recommendations for quality echocardiography laboratory operations. J Am Soc Echocardiogr 2011;24(1):1-10.

[3] Bendick PJ. Scope of practice and clinical standards for the diagnostic medical sonographer. Journal of Diagnostic Medical Sonography 2015;31(4):198-209.

[4] Ramana KV, Kandi S, Boinpally PR. Ethics in medical education, practice and research: an insight. Annals of Tropical Med Public Health 2013;6(6):599-602.

[5] Boyd KM. Medical ethics: principles, persons and perspectives: from controversy to conversation. J Med Ethics 2005;31(8):481-6.

[6] Gillon R, Lloyd A. Principles of health care ethics. London: Routledge \& Kegan Paul 1994.
[7] Centers for Medicare and Medicaid Services. Open Payments. https://www.cms.gov/openpayments/

[8] Hartocollis A. Heart tests at hospital went unread. New York Times May 25, 2010.

[9] Kirkpatrick JN, Pearlman AS. Ethical challenges in the practice of echocardiography: what is right and how do we do it? J Am Soc Echocardiogr 2019;32(2):233-7.

[10] Childress JF, Beauchamp TL. Principles of biomedical ethics. New York: Oxford University Press 2001.

[11] Jonsen AR, Siegler M, Winslade WJ. Clinical ethics: a practical approach to ethical decisions in clinical medicine. New York: Macmillan 1982.

[12] Douglas PS, Khandheria B, Stainback RF, et al. ACCF/ASE/ACEP/ASNC/SCAI/SCCT/SCMR 2007 appropriateness criteria for transthoracic and transesophageal echocardiography: a report of the American College of Cardiology Foundation Quality Strategic Directions Committee Appropriateness Criteria Working Group, American Society of Echocardiography, American College of Emergency Physicians, American Society of Nuclear Cardiology, Society for Cardiovascular Angiography and Interventions, Society of Cardiovascular Computed Tomography, and the Society for Cardiovascular Magnetic Resonance. Endorsed by the American College of Chest Physicians and the Society of Critical Care Medicine. J Am Soc Echocardiogr 2007;20(7):787-805.

[13] Douglas PS, Khandheria B, Stainback RF, et al. ACCF/ASE/ACEP/AHA/ASNC/SCAI/SCCT/SCMR 2008 appropriateness criteria for stress echocardiography: a report of the American College of Cardiology Foundation Appropriateness Criteria Task Force, American Society of Echocardiography, American College of Emergency Physicians, American Heart Association, American Society of Nuclear Cardiology, Society for Cardiovascular Angiography and Interventions, Society of Cardiovascular Computed Tomography, and Society for Cardiovascular Magnetic Resonance: endorsed by the Heart Rhythm Society and the Society of Critical Care Medicine. Circulation 2008;117(11):1478-97.

[14] Orme RMLE, Oram MP, McKinstry CE. Impact of echocardiography on patient management in the intensive care unit: an audit of district general hospital practice. Br J Anaesth 2009;102(3):340-4.

[15] McDonald RW, Rice MJ, Marcella CP, et al. Views of physicians in training on the ethical and legal issues in preliminary reporting of echocardiographic data. J Am Soc Echocardiogr 1991;4(1):1-5. 